\title{
Time-Locked Multi-Color Single-Aperture Fiber Sources via Soliton Self-Mode Conversion
}

\author{
Havva Begüm Kabagöz, Aku Antikainen and Siddharth Ramachandran \\ Boston University, 8 St. Mary's Street, Boston, MA, 02215, USA
}

Multi-color time-synchronized sources are useful for applications such as Raman spectroscopy [1], and midIR generation [2]. Multi-color ultrashort pulse sources from fibers, however, suffer from relative timing-jitter because different colors typically travel at different group velocities, hence experiencing differing levels of RIN to delay variations. Here, we show that the natural intermodal group-velocity locking characteristic of soliton selfmode conversion (SSMC), a process recently discovered in multimode fibers [3, 4], can help generate temporally locked energetic ultrashort pulses across a range of wavelengths, spectrally separated, typically, by one or more Raman Stokes shifts. Control of the wavelength at which the emissions occur, and the number of pulses that can be generated, depends primarily on the group-index separation of the modes in a step-index fiber, which can be controlled via fiber core diameter.

Our pump laser is at 1045-nm with 100-fs pulse width and 1-MHz repetition rate (KMLabs YFi). Spatially sculpting this pump into the $\mathrm{LP}_{0,20}$ mode of a $97-\mu \mathrm{m}$ core diameter, 0.34-NA step-index fiber (Fig. 1.a,b) with an SLM, results in two well-defined $\sim 75$-fs pulses in the $\mathrm{LP}_{0,20}$ and $\mathrm{LP}_{0,19}$ modes, respectively, as they are group velocity matched across one Raman Stokes shift (Fig. 1.c,d). Frequency-doubling the pulses and studying their cross-correlations reveals a jitter of only 13 fs between these two 75-fs pulses, demonstrating temporal locking. The versatility of this technique is illustrated with an identical fiber but now with $70-\mu \mathrm{m}$ core diameter (Fig. 1.e). When the pump is in the $\mathrm{LP}_{0,17}$ mode (Fig. 1.f), three modes are group-index matched across two Raman Stokes shifts (Fig. 1.g) yielding a three-color source (Fig. 1.h) [5]. And, when the pump mode is in the $\mathrm{LP}_{0,14}$ mode instead, four-color emission is obtained (Fig.1.i,j,k).
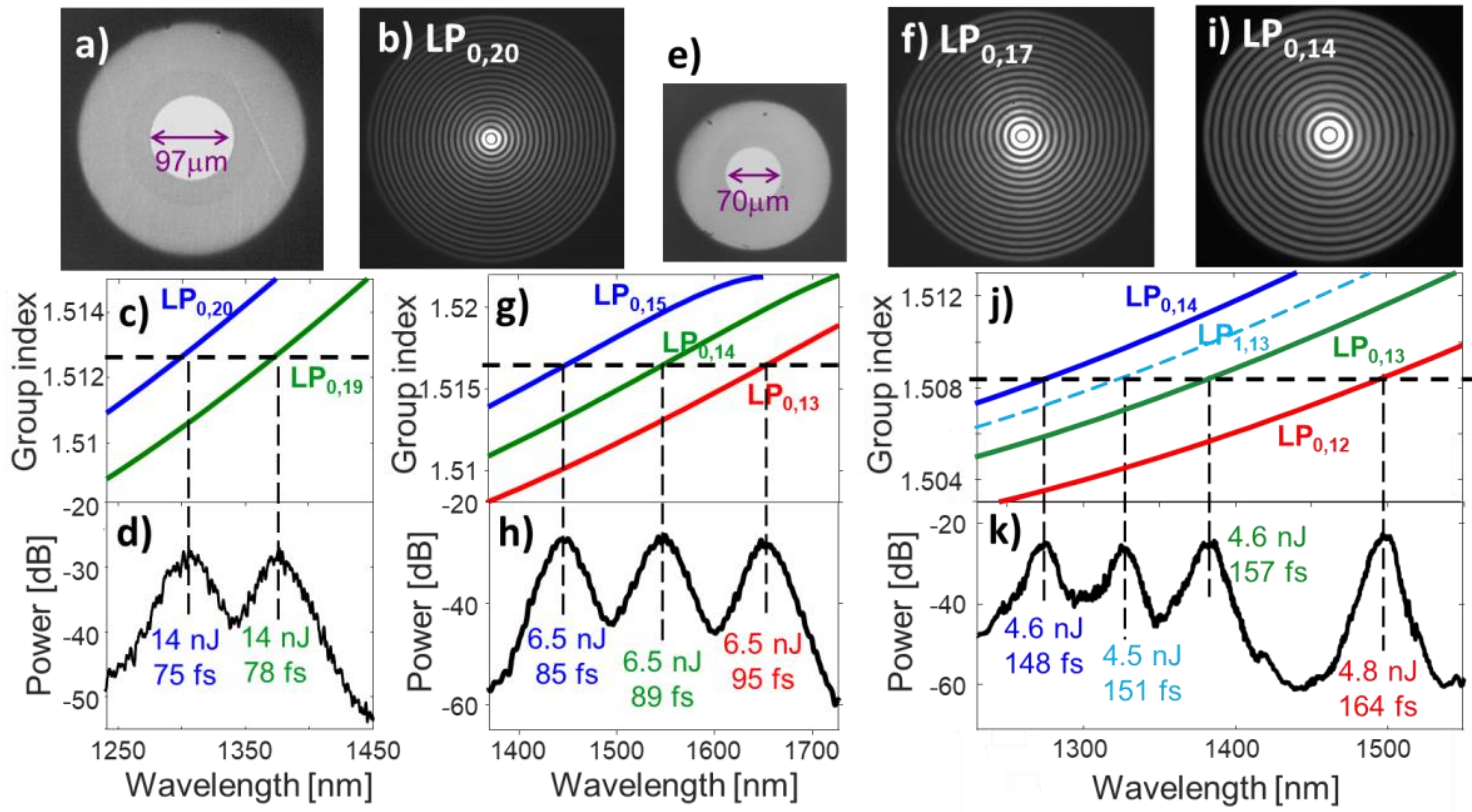

Fig. 1 (a), (e) Facet images of the two fibers used in the experiments. (b), (f), (i) Near field images of the pump modes used in the three experiments. (c), (g), (j) Group-index vs. wavelength for the modes participating in the three respective experiments. (d), (h), (k) the corresponding spectra of the multi-color emissions of the three experiments with pulse energies and autocorrelator-measured pulse widths shown for each peak. Vertical dashed lines show the excellent match between the experimentally observed spectral positions of the pulses, and the corresponding wavelengths at which the modes are group-velocity matched.

Group-velocity matching across spatial modes of a fiber helps obtain multiple 100-fs pulses with up to 14-nJ pulse energies and multiple colors spanning > $200 \mathrm{~nm}$ emitted from a single (fiber) aperture. This is enabled by the process of soliton self-mode conversion, which leverages the new degrees of freedom afforded by multimode nonlinear optics to develop novel ultrashort pulse fiber sources.

[1] M.D. Duncan et al., "Scanning coherent anti-Stokes Raman microscope." Opt. Lett. 7, 350 (1982).

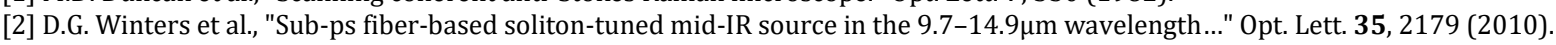

[3] L. Rishøj et al., "Soliton self-mode conversion: revisiting Raman scattering of ultrashort pulses," Optica 6, 304 (2019).

[4] L. Rishøj et al., "Jitter-free, dual-wavelength, ultrashort-pulse, energetic fiber sources using SSMC," Opt. Express 28, 4333 (2020)

[5] H. B. Kabagöz et al., "Time-Synchronized 3-Color Single-Aperture Fiber Sources via SSMC," CLEO, p. SM1P.7 (2020). 